\title{
Vessel Segmentation in Retinal Images
}

\author{
Dietrich Paulus and Serge Chastel* and Tobias Feldmann \\ Institut für Computervisualistik, Universität Koblenz Landau \\ Universitätsstr. 1, 56070 Koblenz, Germany \\ paulus@uni-koblenz.de
}

\begin{abstract}
Detection of the papilla region and vessel detection on images of the retina are problems that can be solved with pattern recognition techniques. Topographic images, as provided e.g. by the HRT device, as well as fundus images can be used as source for the detection. It is of diagnostic importance to separate vessels inside the papilla area from those outside this area. Therefore, detection of the papilla is important also for vessel segmentation. In this contribution we present state of the art methods for automatic disk segmentation and compare their results.

Vessels detected with matched filters (wavelets, derivatives of the Gaussian, etc.) are shown as well as vessel segmentation using image morphology. We present our own method for vessel segmentation based on a special matched filter followed by image morphology. In this contribution we argue for a new matched filter that is suited for large vessels in HRT images.
\end{abstract}

\section{INTRODUCTION}

Glaucoma is the second most common cause of blindness worldwide. ${ }^{1}$ The effects are a damage of the optic nerves; the diagnosis can be based on analysis of the retinal vessels. If not detected in the early stage, damages of the optic nerve are irreversible and can lead to blindness. For diagnostic purposes, automatic vessel segmentation in retinal images is crucial. The vessels can be detected in either reflectivity images (fundus images) or in tomographic images (e.g. HRT images, either reflectivity or topography image).

Vessel structure needs to be described separately for the area inside the optic disk and outside, to increase diagnostic expression.

The paper is organized as follows: In Sect. 2 we discuss the medical importance of image processing on retina images and outline the image acquisition procedure. Deficiencies in image quality can partially be compensated by preprocessing which we describe in Sect. 3. Vessels in the retina need to be analyzed differently for the papilla region and for the rest of the retina. Therefore we show a simple method for papilla detection in Sect. 4. Several methods for vessel segmentation are listed in Sect. 5; some of the methods were originally applied to X-ray images, some to eye images. In this section we propose or new approach to detect vessels in retina images. An evaluation method is proposed in Sect. 6 and we conclude in Sect. 7.

\section{MEDICAL IMPORTANCE OF RETINAL IMAGES}

The optic nerve head is that location where the nerve fibers join and form the optic nerve. The optic nerve head is also called the papilla or the optic disk. As there are no photoreceptors in this area, is also known as the blind spot.

It has been shown that the morphological changes of the optic nerve head precede visual field defects. ${ }^{2}$ Glaucomatous changes are associated with a decreased number of nerve fibers, leading to changes in the configuration of the optic nerve head. Characteristical morphological changes resulting from progressive retinal nerve fiber loss can be described with sectoral morphometric parameters.

Common devices for acquisition of retinal images are fundus cameras that use visible light and CCD cameras to look into the eye. ${ }^{3-8}$ Another option are tomographic imaging techniques which use a confocal scanning laser to reconstruct a three-dimensional tomographic image of the retina; usually, this image volume is then postprocessed to create a $2.5 \mathrm{~d}$ surface image - called the topographic image, and an image that resemples the fudus image - called the reflectivity image (Figure 1). Viewing angle, aperture, etc. differ between devices.

${ }^{*}$ This work was partially funded by E-Eycare, Erlangen, Germany 

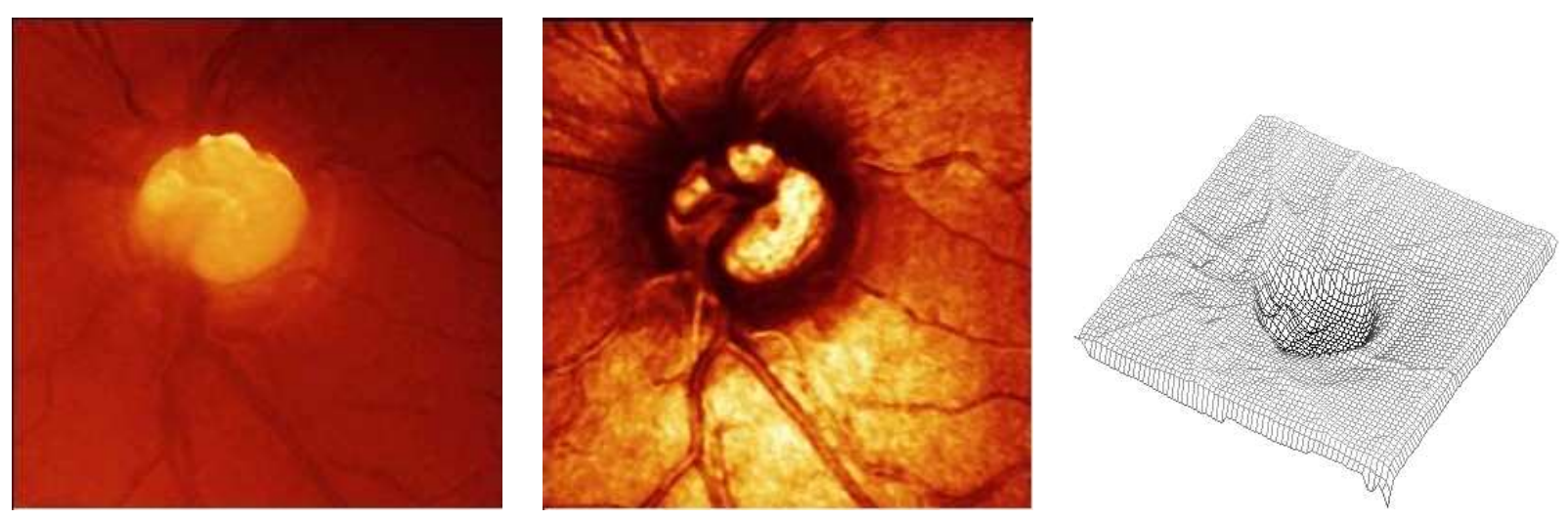

Figure 1. Topographic image, reftectivity image, 3d-topographic image
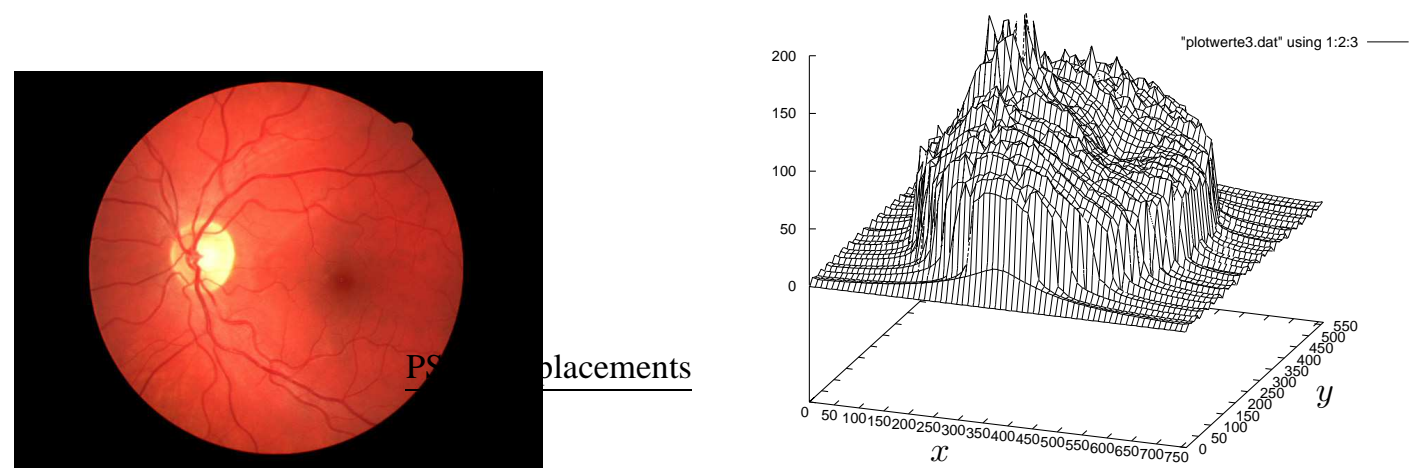

Figure 2. Luminance differences in a fundus image (left), intensity plotted vs. location (right)

\section{SHADING CORRECTION ON RETINAL IMAGES}

As it can be observed in the examples (Figure 2, Figure 3) illumination is not constant accros the image. This is due to the geometry of the acquisition device where illumination and viewing direction are similar but there is a small displacement in the devices.

Several methods for preprocessing of retina images are proposed. ${ }^{9}$ In the following we show methods that eliminate shading effects. Illumination change can be modelled by the following equation: ${ }^{10}$

\section{Luminance}
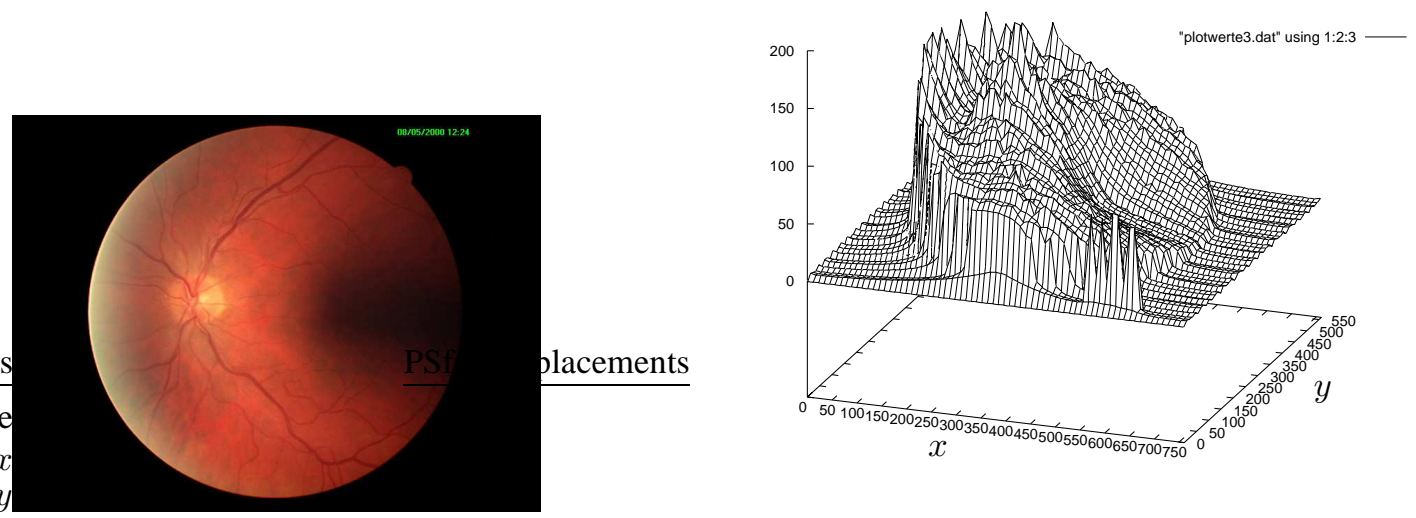

Extreme luminance differences in an image of low quality 


$$
g_{i j}=f_{i j} \gamma_{i j}+\beta_{i j} \quad .
$$

The components $\gamma_{i j}$ and $\beta_{i j}$ are image location dependent multiplicative and additive shading components, respectively, which are generally unknown and need to be estimated. Tomazevic ${ }^{10}$ proposes a scheme for retrospective determination of these parameters. This method has been applied to endoscopic images ${ }^{11}$ : RGB images were converted to YUV, the intensity channel $Y$ was normalized to $Y^{\prime}$ and $Y^{\prime} R G$ was converted back to RGB.

Mean illumination can be locally approximated by a plane in Figure 2 . This can be used for a new strategy. Assuming that the image is homogeneous on a macroscopic scale, we can approximate illumination by a geometric surface approximation. A graph surface used here as a special case of a quadric:

$$
a_{1} j^{2}+a_{2} i^{2}+a_{7} j+a_{8} i+a_{9} f_{i j}+a_{10}=0
$$

where $f_{i j}$ is the value at pixel position $(x, y)$ where we resample to discrete square pixels at $(i, j)$. The distance to this surface at a position $(i, j)$ or $(x, y)$ can then be used to estimate a correction term that reverses the effect of $\gamma_{i j}$ in (1). This formula can also be used to approximate planar surfaces setting $a_{1}$ and $a_{2}$ to zero. Results are shown in Figure 3. For the images in Figure 3 no satisfactory results can be computed; but as medical experts would reject the input images, this failiure is not important.

These algorithms for illumination correction can also be applied to HRT images.
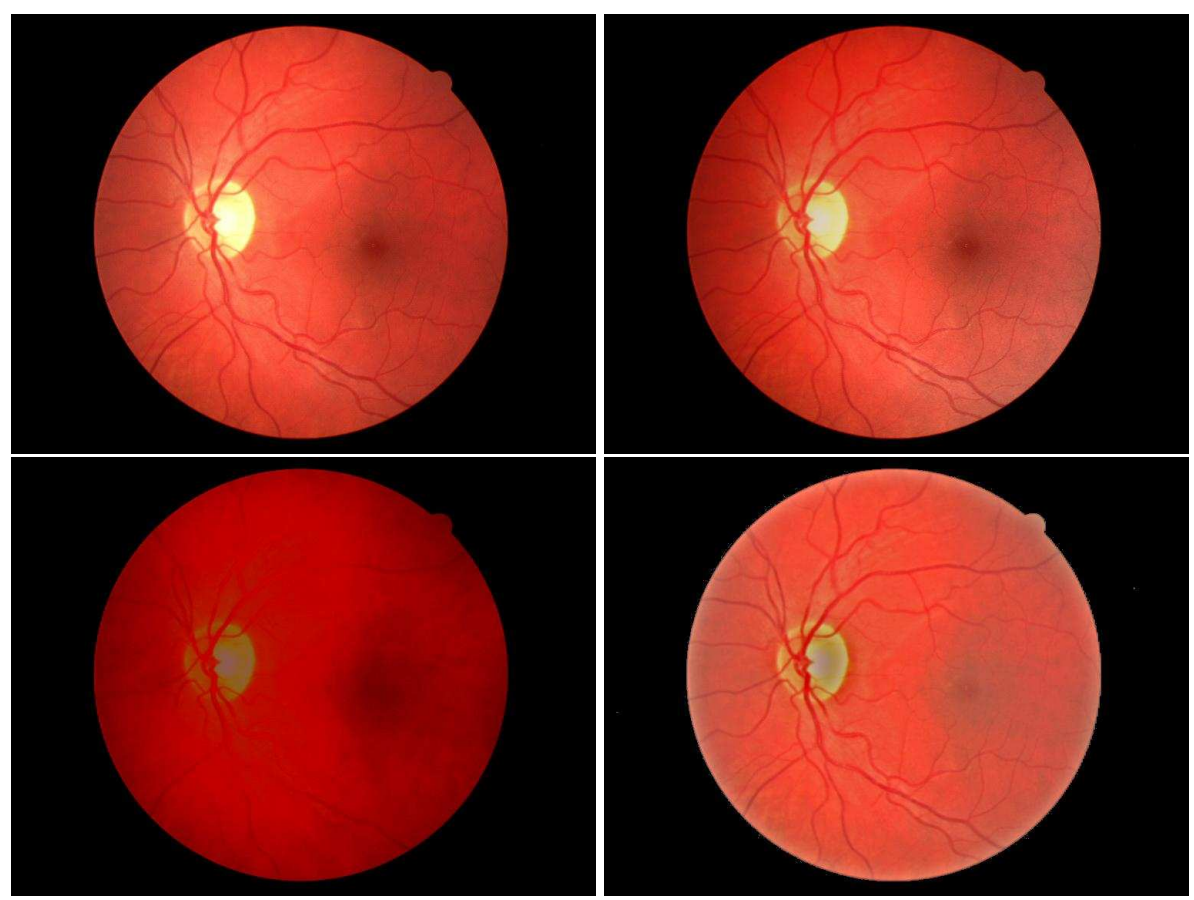

Figure 3. Original image (top left) and corrected images using planar fi t top left, (2) bottom left, homomorphic fi lter bottom right

\section{DETECTION OF THE OPTIC DISK}

Chanwimaluang ${ }^{12}$ uses active contours to approximate the contour of the papilla. Alternative methods based on image morphology have been proposed. ${ }^{9}$

Swindale ${ }^{13}$ approximates the topography by a superposition of a parabola and an exponential function. To determine the parameters, a non-linear optimiziation technique has to be used. 


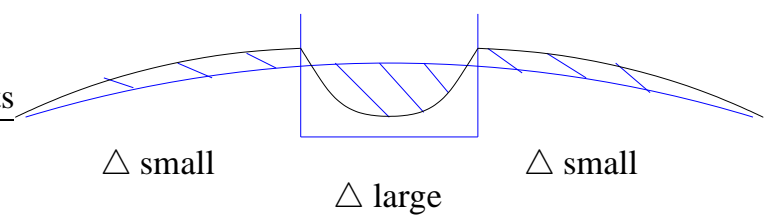

Figure 4. Approximation by parabola

Quadric approximation as in (2) can be applied to the topographic image as outlined in Figure 4 and Figure 5.

Results are shown in Figure 6: absolute difference of the topography image to the approximation outside the papilla region as in Figure 5 yields an estimate of the region of the papilla, as shown in the second line of Figure 6.

\section{VESSEL SEGMENTATION}

Test images that are used in the following are shown in Figure 7. Fang ${ }^{14}$ proposes a two-stage approach to vessel detection, where vessels are found using morpholgy and later vessels are reconstruction. The algorithm works on the green-channel of a fundus image. Results are shown in Figure 8.

Another category of algorithms according to ${ }^{14}$ can be found for matched filters, e.g. Gaussian filters. Results are shown in Figure 9.

The vessels extraction scheme in Chanwimaluang ${ }^{12}$ is based on a matched filter. That filter is based on a simple Gaussian. Since the direction of the vessel is not known, similarly to ${ }^{14}$ the filter is rotated 12 times (every 15 degrees). The filter size is $16 \times 15$ (resolution is not known, images are $605 \times 700$ ). The images are convolved with these filters.

As in Steger's approach ${ }^{15}$ for vessels in radiographs or Pedersen's publication on retinal vessels ${ }^{16}$ we will apply a dedicated detector for vessels of a certain width. Steger ${ }^{15}$ uses a kernel derived from a Gaussian function $G_{\sigma}$

$$
G_{\sigma}(x)=\frac{1}{\sqrt{2 \pi} \sigma} \exp \left(-x^{2} / \sigma^{2}\right)
$$

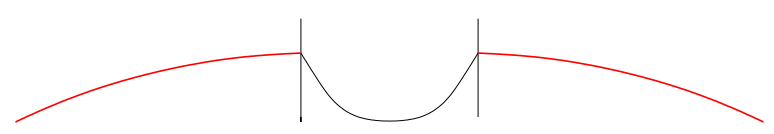

Figure 5. Approximation only outside of papilla region
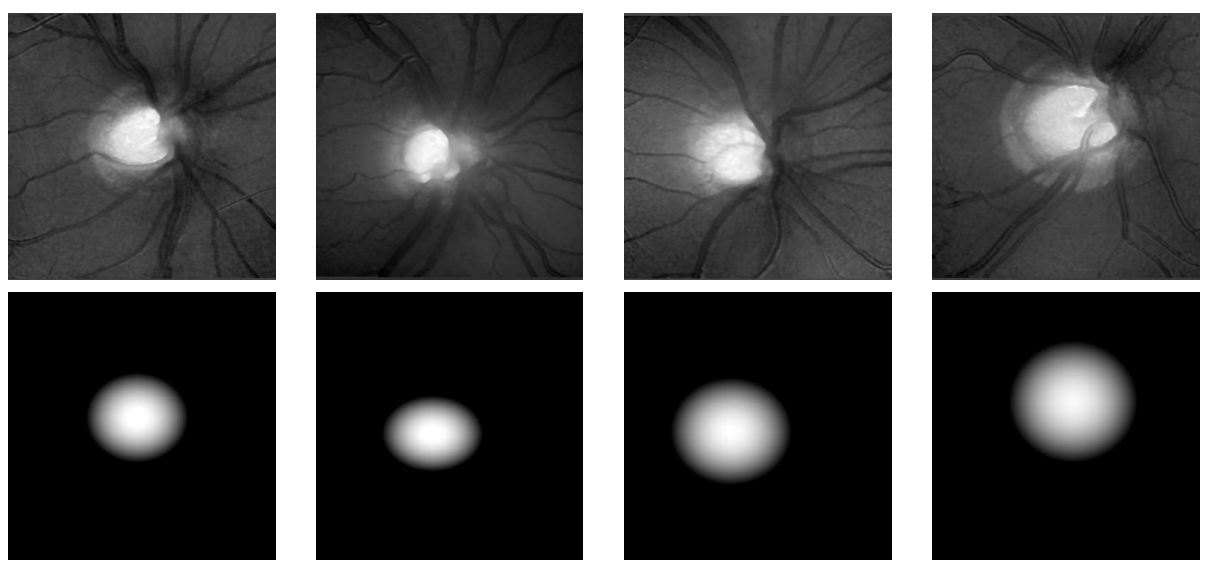

Figure 6. Results of papilla detection 

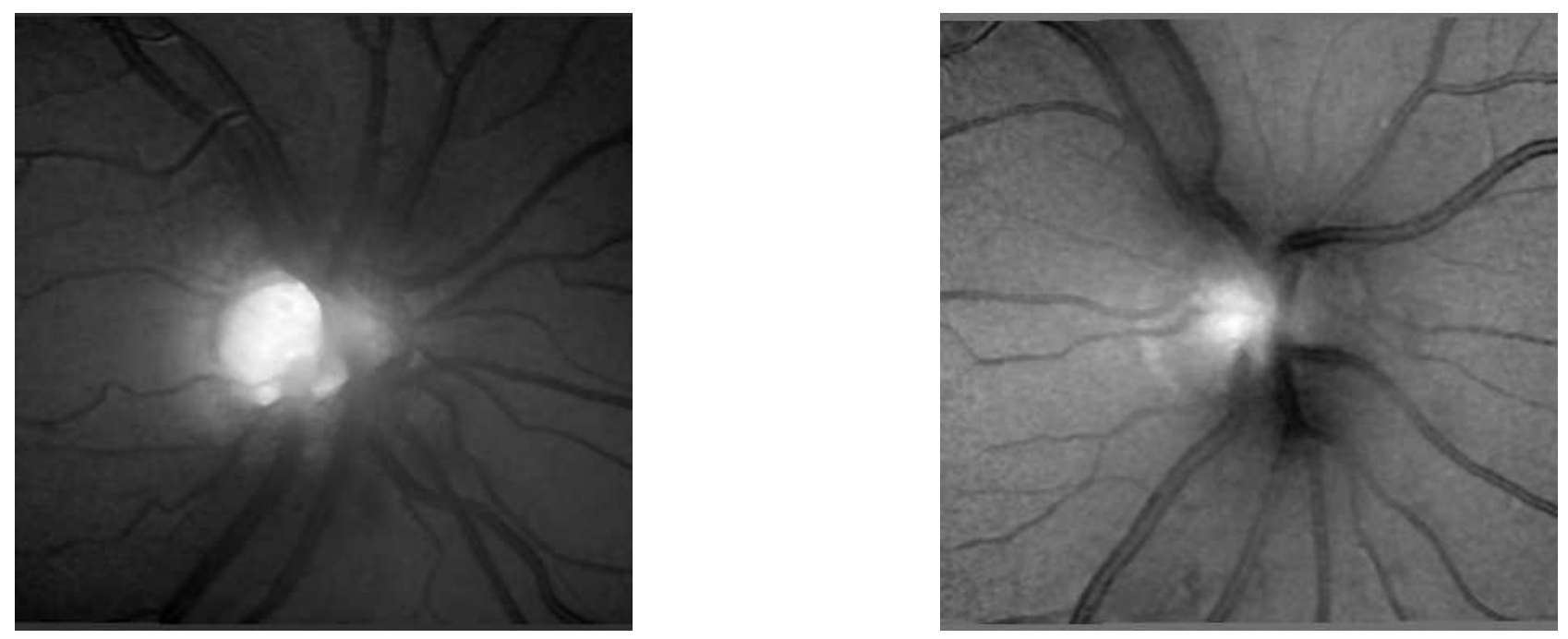

Figure 7. Test HRT images of different quality

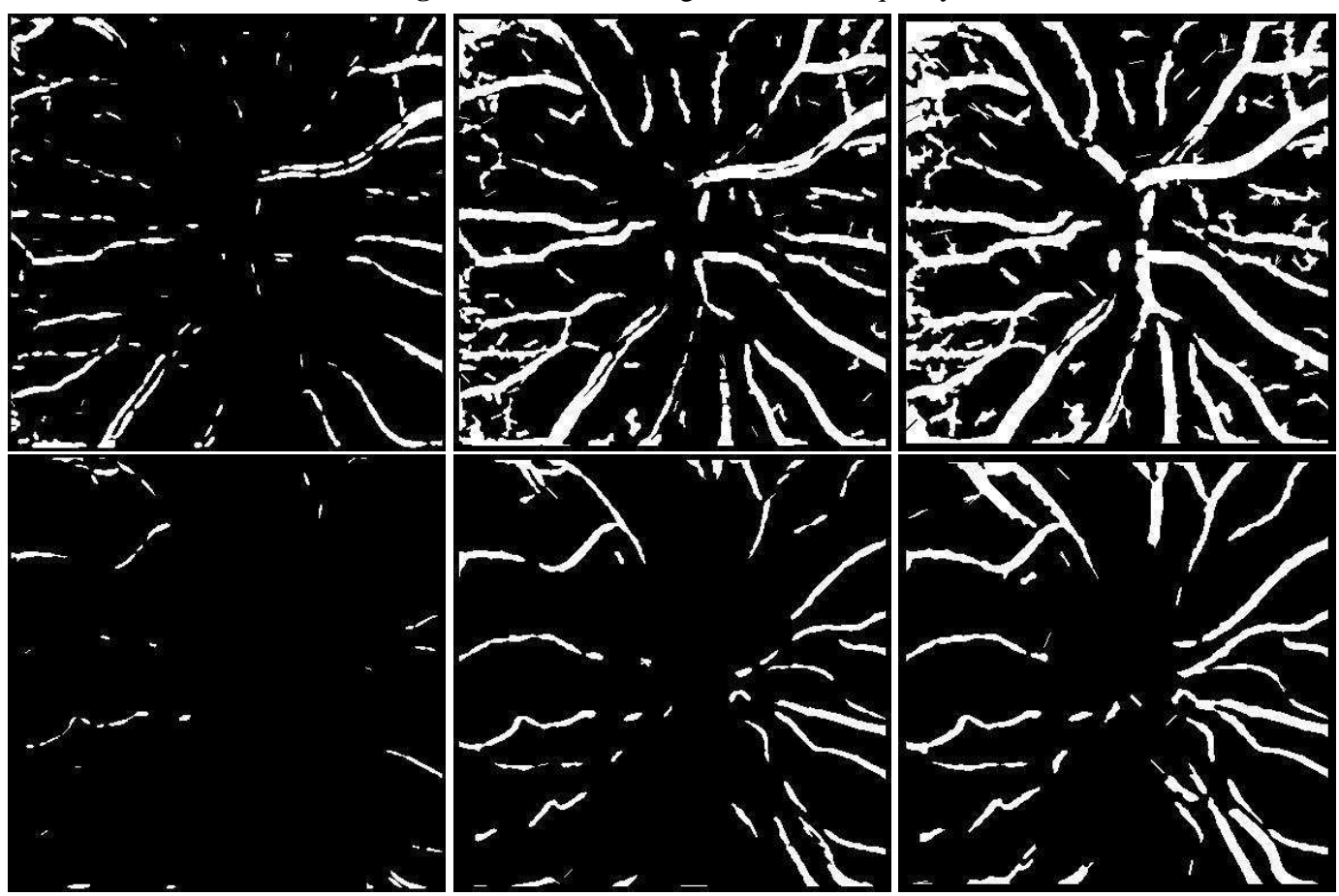

Figure 8. Some segmentation results of Fang's algorithms ${ }^{14}$ on the images in Figure 7. From the left to the right, the size of the morphological element is respectively 7, 9, and 11. The left image is under-segmented, the right one is over-segmented. Top row: hrt image, bottom row fundus image (Figure 7).

$$
G_{\sigma(x)}^{\prime}=\frac{-x}{\sqrt{2 \pi} \sigma^{3}} e^{-\frac{x^{2}}{2 \sigma^{2}}}
$$

to detect bar-shaped vessels (6) as in Figure 10. Convolution of the image with the first derivative of the Gaussian (4) will detect the center line of vessels of width $w=\sqrt{3} \sigma$ at the zero crossing of the convolution result, as exemplified in 

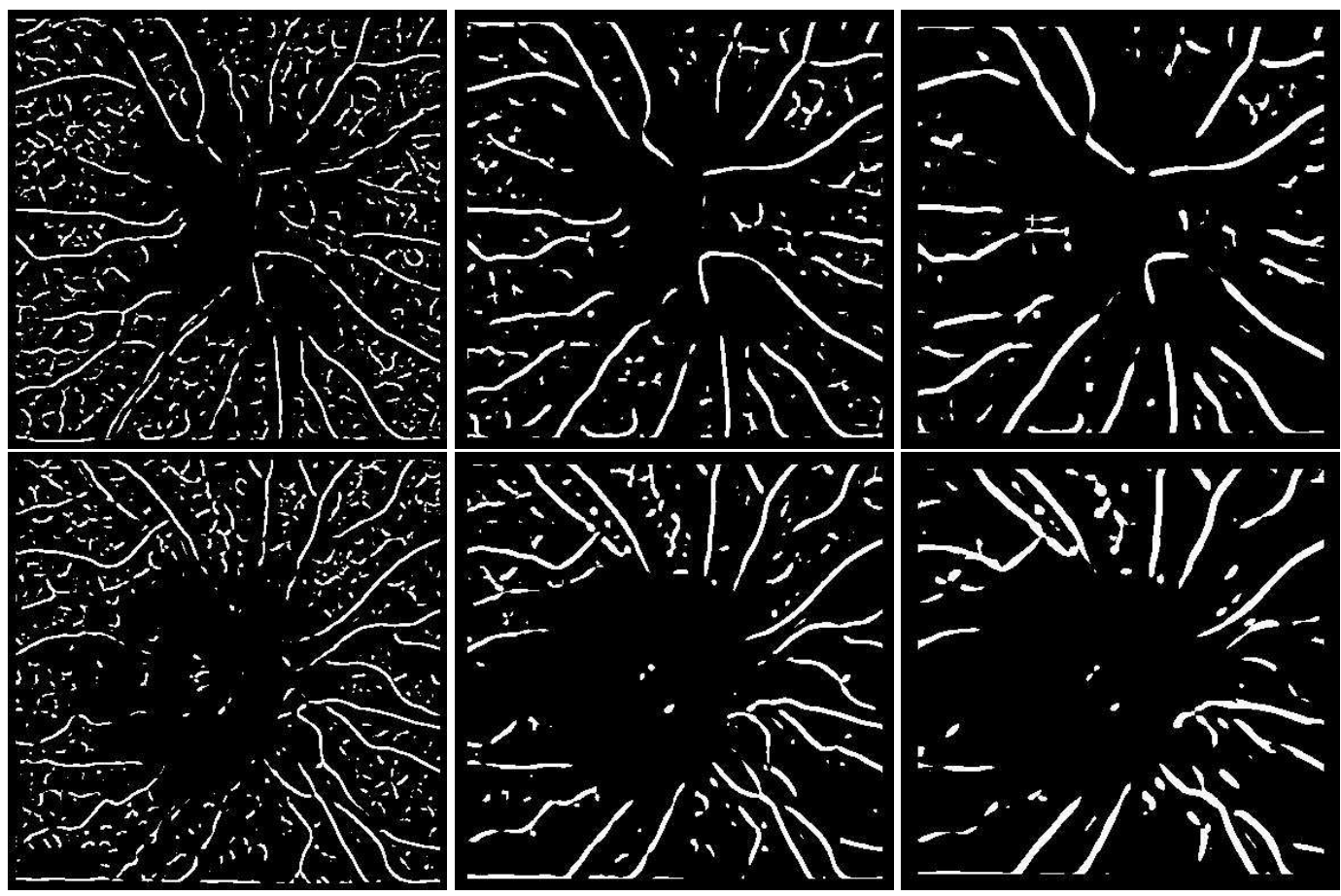

Figure 9. Some segmentation results of Chanwimaluang's algorithm. ${ }^{12}$ on the images in Figure 7.

Figure 11 for the following functions:

$$
\begin{aligned}
r_{b}(x, \sigma, w, h) & =G_{\sigma}(x) * f_{\mathrm{bar}}(x) \\
r_{b}^{\prime}(x, \sigma, w, h) & =G_{\sigma}^{\prime}(x) * f_{\mathrm{bar}}(x) \\
r_{b}^{\prime \prime}(x, \sigma, w, h) & =G_{\sigma}^{\prime \prime}(x) * f_{\mathrm{bar}}(x)
\end{aligned}
$$

The width of the filter thus has to be set much wider than for normal edge detection - e.g. for Canny edge detection, as the filter needs to find the left and right part of a bar shaped edge. The centerline of the vessel is found at the zero-crossing $x_{0}$ of $r_{b}^{\prime}(x, \sigma, w, h)$. The vessel strength is derived from the value of $r_{b}^{\prime \prime}\left(x_{0}, \sigma, w, h\right)$. Parameters of the algorithm are the width of the vessel, the intensity of the background, and the contrast. Results are shown in Figure 12 and in Figure 13.

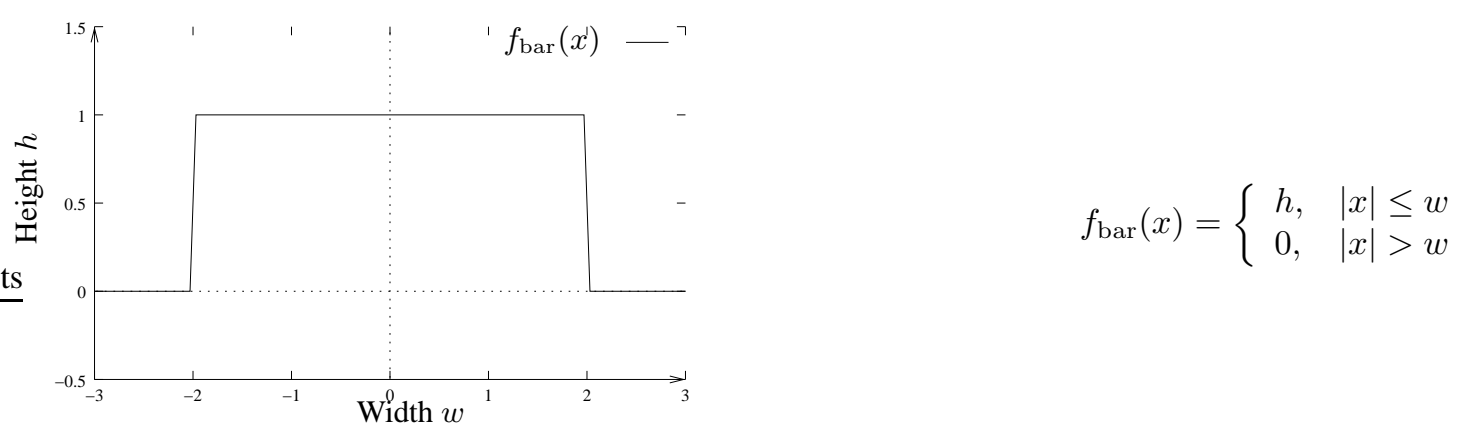

Figure 10. Example $(h=1, w=2)$ and mathematical model for bar-shaped profi le.

For model-based analysis Wang ${ }^{17}$ proposes a similar idea where the choice of parameters is partially solved by an optimization of the coefficients of rotated Gaussian convolution kernels. 

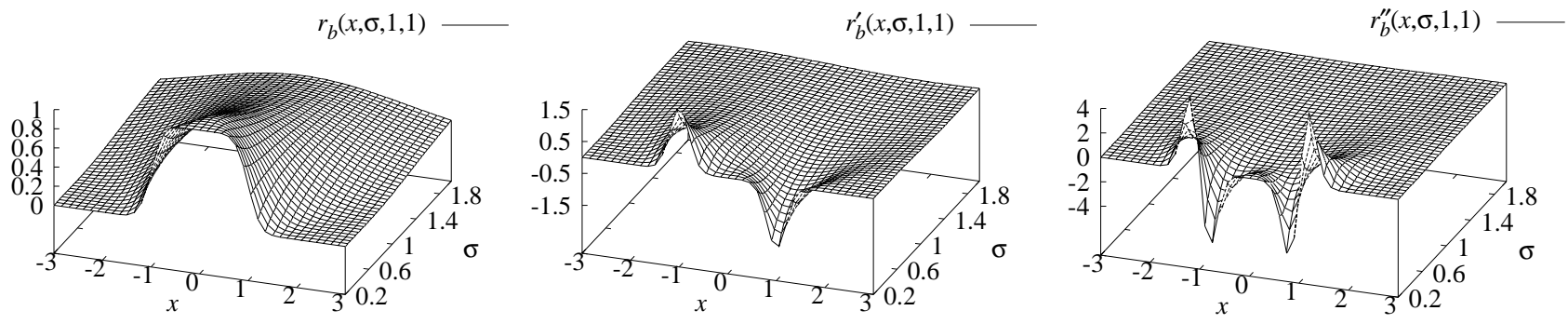

Figure 11. Bar-shaped profi le $f_{\mathrm{b} a r}$ convolved with derivatives of Gaussian for $x \in[-3,3]$ and $\sigma \in[0.2,1.8]$.

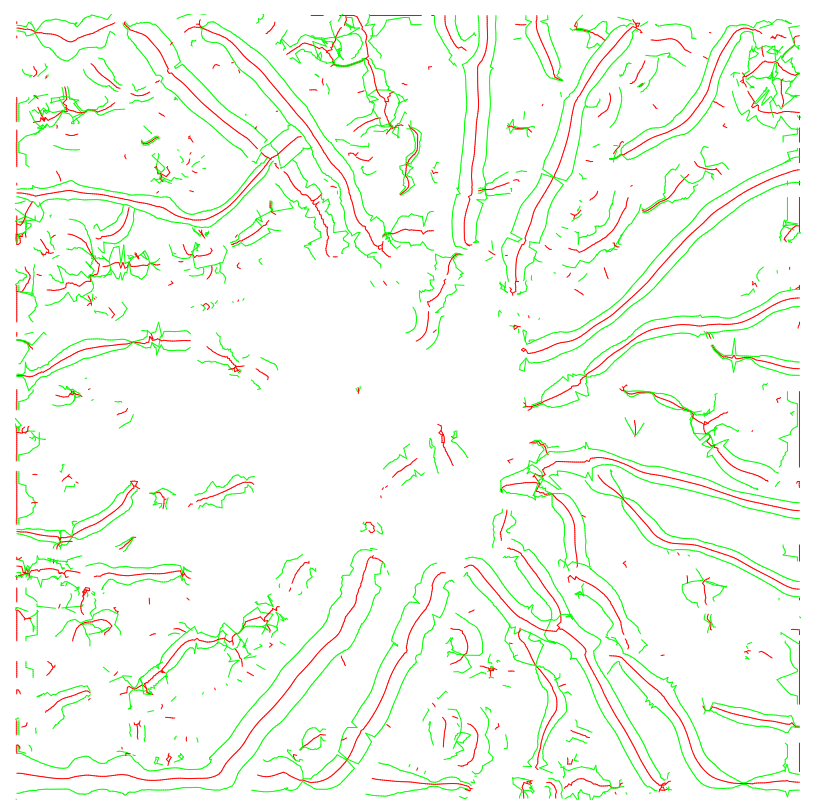

Figure 12. Steger's algorithm ${ }^{15}$ on Figure 7 left

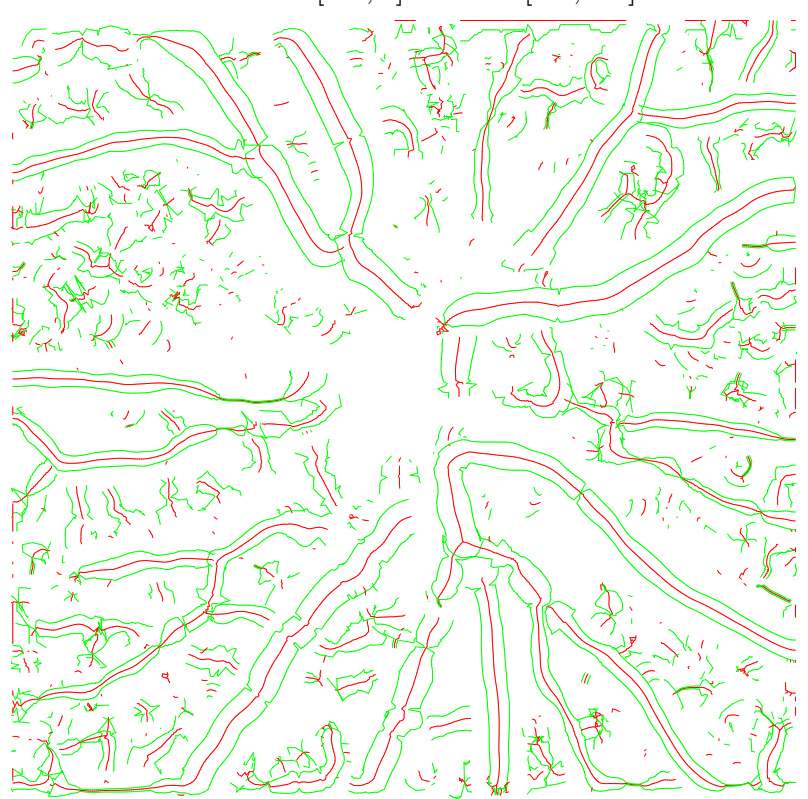

Figure 13. Steger's algorithm on Figure 7 right

The kernel has the following form:

$$
G(\omega)=\sum_{i=1}^{M}\left|G_{m}(\omega) \exp \left[-j \phi_{m}(\omega)\right]\right|
$$

where $G_{m}$ is a Gaussian, $\phi_{m}$ is a phase function (i.e. $G_{m}$ is rotated).

If we have a careful look at Figure 14 we realize that the wide retinal vessels have typical double-edge shape in HRT images. This also happens sometimes for fundus images. Figure 14 right shows the input data, filtered input data and a suitable DOG mask

$$
H_{\mathrm{x}}(x)=G_{\sigma_{1}}(x)-G_{\sigma_{2}}(x)
$$

These vessels exhibit a highlight in the center of the vessels which causes two extra edges in parallel to the vessels outline. The DOG detector optimally fits this behaviour. Alternatively, the second derivative of (3) can be chosen. While Steger ${ }^{15}$ convolves the input image with the second derivative of the Gaussian (cmp. Figure 11 right), we now need to find the maximum correlation of the DOG filter with the input image. Vessels can now be found matching rotated versions of the mask with the input image. We chose discrete orientations at angles 30, 45, 60, 90, 120,135, 150, 180 degrees and compute the sum of squared differences to the input region of interest. For each position $(i, j)$ in the input image we select the mask with the maximum similarity. Its direction is recorded in the orientation part of an edgel, the strength is set to the maximum response. This results in an edge image that can be subject to line detection, e.g. using hysteresis threshold 

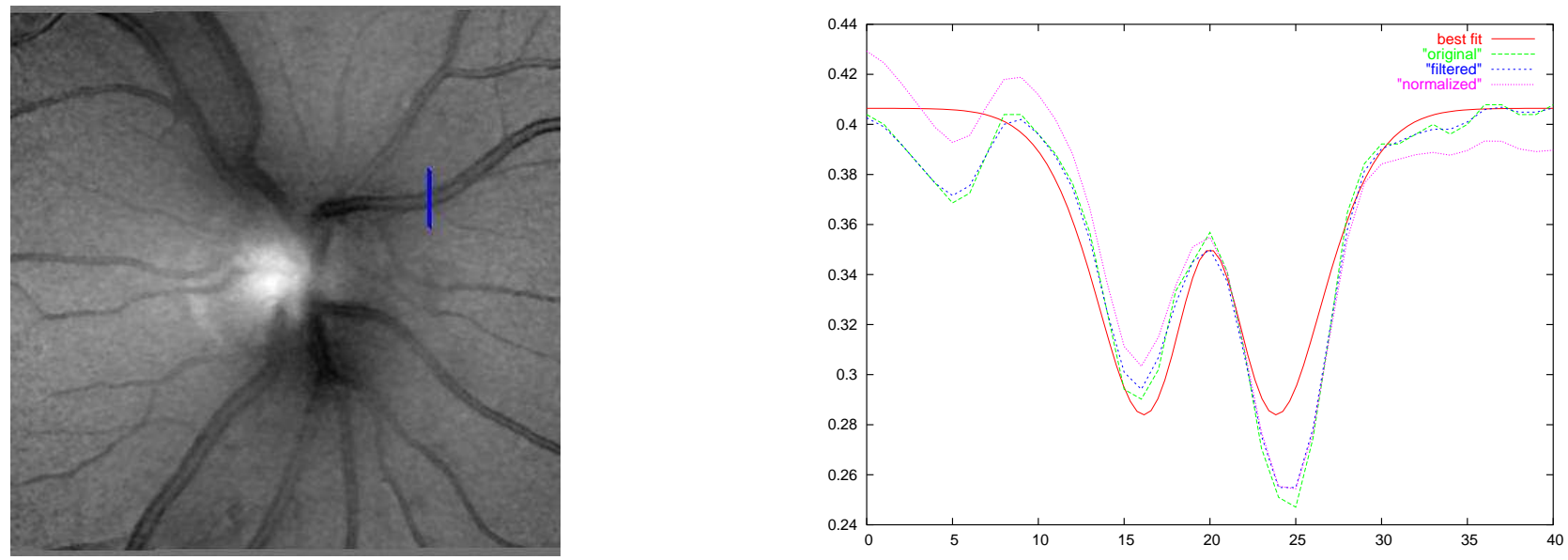

Figure 14. Cross section in HRT image (marked in the image left)

searching. The strength computation on the test images are shown in Figure 15 where we used the second derivative of the Gaussian with $\sigma=3.5$. Line detection is shown in Figure 16 right.
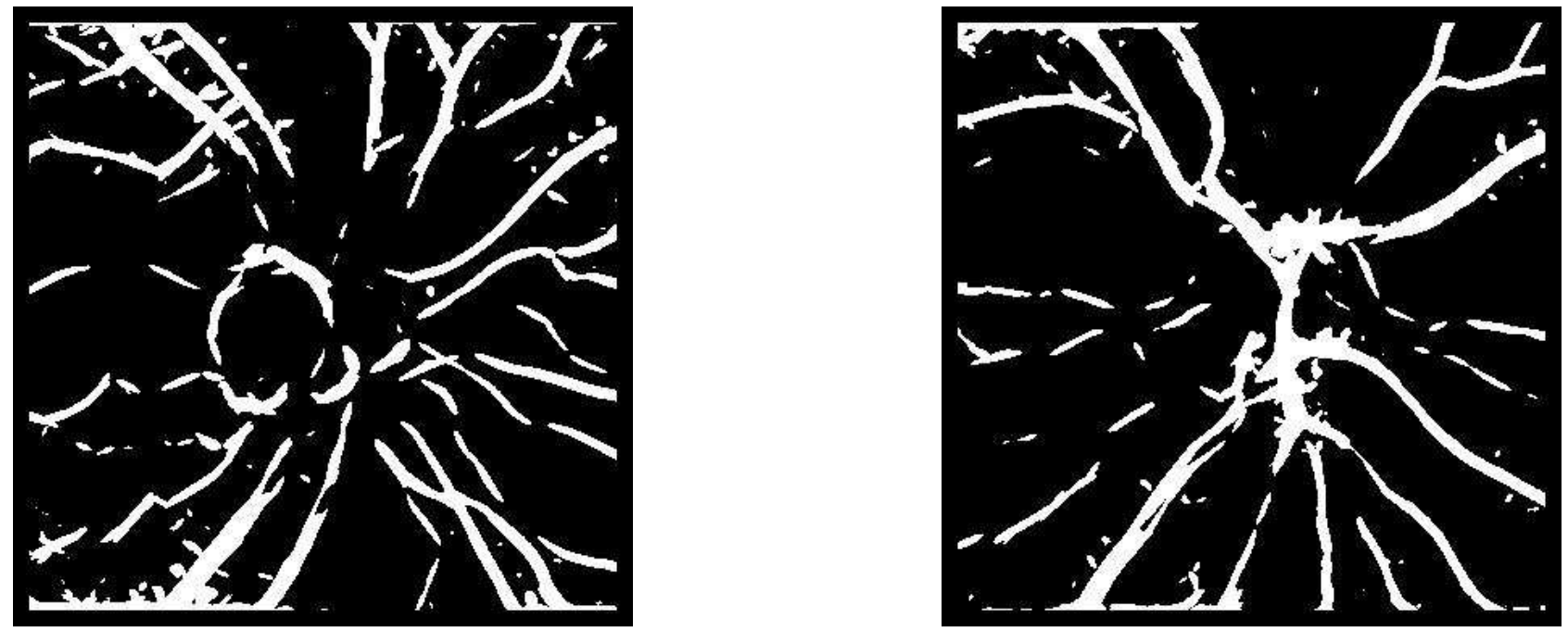

Figure 15. Result on test images

\section{EVALUATION METHODS FOR VESSEL SEGMENTATION}

In order to evaluate a segmentation method, measures for the quality of the results are required. Such measures are proposed e.g. by Förstner ${ }^{18}$ and Haralick. ${ }^{19}$ Using a penalty term for the distance of the segmented vessel to the correct position, the accuracy of the detection can be judged. In addition, missing vessel segments need to be penalized as well.

The important problem is to have a comparison to a gold standard for a given segmentation, no matter which distance measures and penalty terms are used. For vessel segmentation, we can either hand-segment an image, use a phantom with well-known geometry and segment its images, or use synthetic images with well-known structure, i.e. synthetic vessel trees, optionally distorted by noise. Vessel trees were synthesized based on real retinal images using a grammar and evolutionary algorithms. ${ }^{20}$ For our experiments we manually analyzed several images with respect to distances between junctions, and width and created a statistical model. From the initial vessel tree a double-edge vessel is created by simple image morpholgy; this image is smoothed and corrupted by Gaussian noise. Figure 16 shows an example. 

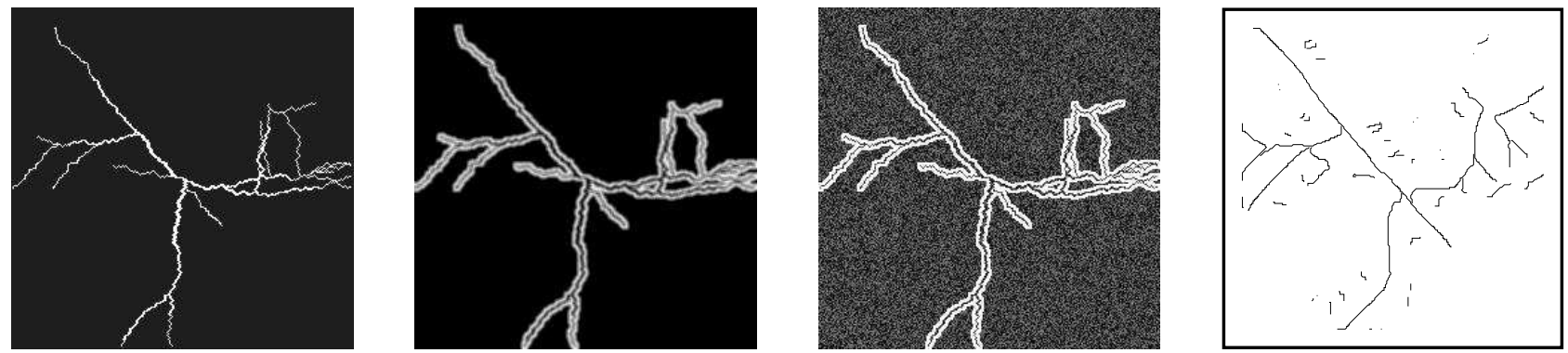

Figure 16. Synthetic Vessel tree, synthetic retinal image, synthetic retinal image with noise, result of segmentation

\section{CONCLUSION AND FURTHER WORK}

We have shown by examples that an correlation of a specific vessel template can be used to detect double-edge vessels in retinal images. The evaluation methods proposed in Sect. 6 have not been applied, yet, to a data base. This would require a manual segmentation of the images. It is planned to compare different segmentation algoriths on synthetic vessel trees.

It is also planned to derive further features from the vessel structure and to use these to train a classifier. The Erlangen Glaucoma Registry currently consists of anamnestic and diagnostic data from repeated observations of 1100 subjects starting 12 years ago. ${ }^{21,22}$ It is planned to use this data set for glaucoma classification on vessel features.

\section{Acknowledgments}

The synthetic vessels were created by a program written by Csaba Kertez who is funded by an Erasmus grant. ${ }^{\dagger}$ Thanks to Dag Ewering for the discussion on vessel filters. The approximation of the topographic image was realized by Katharina Brass. Shading correction was implemented by Annika Hirsch. Thanks to Radim Chrástek and Dr. G. Michelson for many fruitful discussions on retinal images.

\section{REFERENCES}

1. H. A. Quigley, "Number of people with glaucoma worldwide," British Journal of Ophthalmology 80, pp. 389-393, 1996.

2. H. A. Quigley, J. Katz, R. J. Derick, D. Gilbert, and A. Sommer, "An evaluation of optic disc and nerve fiber layer examinations in monitoring progression of early glaucoma damage," Ophthalmology 99(1), pp. 19-28, 1992.

3. F. S. Mikelberg, C. M. Parfitt, N. V. Swindale, S. L. Graham, S. M. Drance, and R. Gosine, "Ability of the Heidelberg Retina Tomograph to detect early glaucomatous visual field loss," Journal of Glaucoma 4, pp. 242-247, 1995.

4. M. Iester, J. B. Jonas, C. Y. Mardin, and W. M. Budde, "Discriminant analysis models for early detection of glaucomatous optic disc changes.," British Journal of Ophthalmology 84(5), pp. 464-8, 2000.

5. C. Y. Mardin, F. Horn, W. M. Budde, and J. B. Jonas, "Monitoring of morphometric changes of optic discs with morphologic progression of glaucomatous optic atrophy by means of laser scanner tomography," Klinische Monatsblätter für Augenheilkunde 217(2), pp. 82-87, 2000.

6. B. C. Chauhan, T. A. McCormick, M. T. Nicolela, and R. P. LeBlanc, "Optic disc and visual field changes in a prospective longitudinal study of patients with glaucoma: Comparison of scanning laser tomography with conventional perimetry and optic disc photography," Archives of Ophthalmology 119(10), pp. 1492-1499, 2001.

7. C. Y. Mardin and A. G. Jünemann, "The diagnostic value of optic nerve imaging in early glaucoma," Current Opinion in Ophthalmology 12(2), pp. 100-104, 2001.

8. M. J. Greaney, D. C. Hoffmann, D. F. Garway-Heath, M. Nakla, A. L. Coleman, and J. Caprioli, "Comparison of optic nerve imaging methods to distinguish normal eyes from those with glaucoma," Investigative Ophthalmology and Visual Science 43(1), pp. 140-145, 2002.

\footnotetext{
${ }^{\dagger}$ The program is available in the PUMA environment, ${ }^{23}$ http: //www . uni-koblenz . de/puma
} 
9. R. Chrástek, M. Wolf, K. Donath, H. Niemann, D. Paulus, T. Hothorn, B. Lausen, R. Lämmer, C. Y. Mardin, and G. Michelson, "Automated segmentation of the optic nerve head for diagnosis of glaucoma," Medical Image Analysis , p. to appear, 2005.

10. D. Tomazevic, B. Likar, and F. Pernus, "A comparison of retrospective shading correction techniques," in International Conference on Pattern Recognition (ICPR'00), 3, pp. 564-567, IEEE Inc., 2000.

11. C. Münzenmayer, F. Naujokat, S. Mühldorfer, and T. Wittenberg, "Enhancing texture analysis by color shading correction," in 9. Workshop Farbbildverarbeitung, K.-H. Franke, ed., Zentrum für Bild- und Signalverarbeitung e.V., (Ilmenau), 2003.

12. T. Chanwimaluang and G. Fan, "An efficient algorithm for extraction of anatomical structures in retinal images," in Proc. IEEE International Conference on Image Processing, pp. 1093-1096, (Barcelona, Spain), 92003. http://www.vcipl.okstate.edu.

13. N. V. Swindale, G. Stjepanovic, A. Chin, and F. S. Mikelberg, "Automated analysis of normal and glaucomatous optic nerve head topography images," Investigative Ophthalmology and Visual Science 41, pp. 1730-1742, 62000.

14. B. Fang, W. Hsu, and M. Lee, "Reconstruction of vascular structures in retinal images," in ICIP '03, pp. 157-160, 2003.

15. C. Steger, "An unbiased detector of curvilinear structures," Pattern Analysis and Machine Intelligence 20(2), pp. 113-125, 1998.

16. L. Pedersen, M. Grunkin, B. Ersbøll, K. Madsen, M. Larsen, N. Christofferson, and U. Skands, "Quantitative measurement of changes in retinal vessel diameter in ocular fundus images," Pattern Recognition Letters 21, pp. 12151223, Aug. 2000.

17. L. Wang and A. H. Bhalerao, "Model based segmentation for retinal fundus images," in SCIA03, pp. 422-429, 2003.

18. W. Förstner, "Diagnostics and performance evaluation in computer vision," Performance versus Methodology in Computer Vision, NSF/ARPA Workshop, Seattle, pp. 11-24, 1994.

19. R. M. Haralick, "Performance characterization in computer vision," Computer Vision, Graphics, and Image Procssing 60, pp. 245-249, September 1994.

20. G. Kókai, Z. Tóth, and R. Ványi, "Modelling blood vessel of the eye with parametric L-systems using evolutionary algorithms," in Artificial Intelligence in Medicine, Proceedings of the Joint European Conference on Artificial Intelligence in Medicine and Medical Decision Making, AIMDM'99, W. Horn, Y. Shahar, G. Lindberg, S. Andreassen, and J. Wyatt, eds., Lecture Notes of Computer Science 1620, pp. 433-443, Springer-Verlag, (Aalborg, Denmark), 20-24 June 1999.

21. C. Y. Mardin and F. K. Horn, "Influence of optic disc size on the sensitivity of the Heidelberg Retina Tomograph," Graefe's Archive for Clinical and Experimental Ophthalmology 236(9), pp. 641-645, 1998.

22. G. Michelson and M. J. M. Groh, "Screening models for glaucoma," Current Opinion in Ophthalmology 12(2), pp. 105-111, 2001.

23. S. Chastel, G. Schwab, and D. Paulus, "Web interface for image processing algorithms," in Internet Imaging V, S. Santini and R. Schettini, eds., The International Society for Optical Engeneering, (San Jose), 12004 . volume 5304 ISBN 0-8194-5207-6. 